\title{
NONCOMMUTATIVE SUPERSYMMETRIC THEORIES
}

\author{
Victor O. Rivelles* \\ Instituto de Física \\ Universidade de São Paulo \\ Caixa Postal 66318, 05315-970, São Paulo, SP, Brazil \\ E-mail: rivelles@fma.if.usp.br
}

ABstract: We discuss the renormalization properties of noncommutative non-gauge supersymmetric field theories.

\section{Introduction}

The main feature of noncommutative field theories is the ultraviolet (UV) and infrared (IR) mixing of divergences [i] [1] $y,[x, y]=\theta$, at the quantum level entails an uncertainty $\Delta x \Delta y \sim \theta$. Together with the usual uncertainty relation $\Delta x \Delta p_{x} \sim 1$ we find that $\Delta y \sim \theta \Delta p_{x}$, which means that the UV regime in the $x$-direction produces an IR effect in the $y$-direction and vice-versa. At the field theory level this phenomenon manifests itself as a mixture of UV and IR divergences already at the one loop level [2]. If we choose to renormalize the theory in the usual way then the remaining IR divergence becomes a source of trouble since it leads, in general, to non-integrable divergences in higher loop orders jeopardizing renormalizability. It was then suggested that a possible way out would be the introduction of supersymmetry [3i]. Since supersymmetric theories have only logarithmic divergences it could be possible that the dangerous UV/IR mixing could be absent. This was shown at one loop level for the two-point function of the gauge field [i] general was still lacking. Then the noncommutative Wess-Zumino model was shown to be free of UV/IR mixing to all loop orders [i5] providing the first noncommutative field theory which is fully renormalizable in four dimensions. Its low energy properties were studied in detail [6] $\left[\bar{b}^{-}\right.$. Other noncommutative supersymmetric non-gauge theories were also found to be free of UV/IR mixing. For instance, the supersymmetric nonlinear sigma model in three dimensions turns out to be renormalizable in the $1 / N$ expansion [i]

\footnotetext{
${ }^{*}$ Speaker.
} 
Spontaneous symmetry breaking also has troubles in the presence of noncommutativity

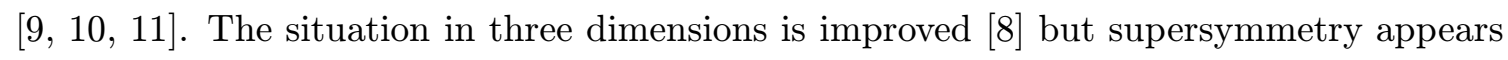
to play no role in this case. Thus, supersymmetry seems to be essential for renormalizability in noncommutative field theories. However, this turns out to be true only for non-gauge theories. Supersymmetric gauge theories are still under intense scrutiny.

\section{Noncommutative Scalar Field Theory}

Noncommutative field theories are obtained from the commutative ones just by replacing the ordinary field multiplication by the Moyal product defined as

$$
\left(\phi_{1} \star \phi_{2}\right)(x) \equiv\left[e^{i \frac{1}{2} \theta^{\mu \nu} \frac{\partial}{\partial x^{\mu}} \frac{\partial}{\partial y^{\nu}}} \phi_{1}(x) \phi_{2}(y)\right]_{y=x} .
$$

Then the noncommutative $\phi^{4}$ model in $3+1$ dimensions reads as [2]

$$
S=\int d^{4} x\left(\frac{1}{2} \partial_{\mu} \phi \star \partial^{\mu} \phi-\frac{m^{2}}{2} \phi \star \phi-\frac{g^{2}}{4 !} \phi \star \phi \star \phi \star \phi\right) .
$$

We now proceed in the standard way. The quadratic terms gives the propagator. Since the Moyal product has the property

$$
\int d x(f \star g)(x)=\int d x(g \star f)(x)=\int d x f(x) g(x),
$$

the propagator is the same as in the commutative case. This is a general property of noncommutative theories: the propagators are not modified by the noncommutativity. The vertices, however, are in general affected by phase factors. In the present case we get, in momentum space,

$$
\begin{aligned}
& -\frac{g^{2}}{2} \int d^{4} x \phi \star \phi \star \phi \star \phi=-\frac{g^{2}}{6} \int d k_{1} d k_{2} d k_{3} d k_{4} \delta\left(k_{1}+k_{2}+k_{3}+k_{4}\right) \times \\
& {\left[\cos \left(\frac{1}{2} k_{1} \wedge k_{2}\right) \cos \left(\frac{1}{2} k_{3} \wedge k_{4}\right)+\cos \left(\frac{1}{2} k_{1} \wedge k_{3}\right) \cos \left(\frac{1}{2} k_{2} \wedge k_{4}\right)+\right.} \\
& \left.\cos \left(\frac{1}{2} k_{1} \wedge k_{4}\right) \cos \left(\frac{1}{2} k_{2} \wedge k_{3}\right)\right] \phi\left(k_{1}\right) \phi\left(k_{2}\right) \phi\left(k_{3}\right) \phi\left(k_{4}\right) .
\end{aligned}
$$

We can now compute the one loop correction for the two-point function. It is easily found to be

$$
\frac{g^{2}}{3(2 \pi)^{4}} \int d^{4} k\left(1+\frac{1}{2} \cos (k \wedge p)\right) \frac{1}{k^{2}+m^{2}} .
$$

The first term is the usual one loop mass correction of the commutative theory (up to a factor $1 / 2$ ) which is quadratically divergent. The second term is not divergent due to the oscillatory nature of $\cos (k \wedge p)$. This shows that the nonlocality introduced by the Moyal product is not so bad and leaves us with the same divergence structure of the commutative theory. This is also a general property of noncommutative theories [i] 1 in $]$. To take into account the effect of the second term we regularize the integral using the Schwinger parametrization

$$
\frac{1}{k^{2}+m^{2}}=\int_{0}^{\infty} d \alpha e^{-\alpha\left(k^{2}+m^{2}\right)} e^{-\frac{1}{\Lambda^{2} \alpha}}
$$


where a cutoff $\Lambda$ was introduced. We find

$$
\Gamma^{(2)}=\frac{g^{2}}{48 \pi^{2}}\left[\left(\Lambda^{2}-m^{2} \ln \left(\frac{\Lambda^{2}}{m^{2}}\right)+\ldots\right)+\frac{1}{2}\left(\Lambda_{e f f}^{2}-m^{2} \ln \left(\frac{\Lambda_{e f f}^{2}}{m^{2}}\right)+\ldots\right)\right],
$$

where

$$
\Lambda_{e f f}^{2}=\frac{1}{\frac{1}{\Lambda^{2}}+\tilde{p}^{2}}, \quad \tilde{p}^{\mu}=\theta^{\mu \nu} p_{\nu}
$$

Note that when the cutoff is removed, $\Lambda \rightarrow \infty$, the noncommutative contribution remains finite providing a natural regularization. Also $\Lambda_{\text {eff }}^{2}=\frac{1}{\tilde{p}^{2}}$ which diverges either when $\theta \rightarrow 0$ or when $\tilde{p} \rightarrow 0$.

The one loop effective action is then

$$
\int d^{4} p \frac{1}{2}\left(p^{2}+M^{2}+\frac{g^{2}}{96 \pi^{2}\left(\tilde{p}^{2}+1 / \Lambda^{2}\right)}-\frac{g^{2} M^{2}}{96 \pi^{2}} \ln \left(\frac{1}{M^{2}\left(\tilde{p}^{2}+1 / \Lambda^{2}\right)}\right)+\ldots\right) \phi(p) \phi(-p),
$$

where $M$ is the renormalized mass. Let us take the limits $\Lambda \rightarrow \infty$ and $\tilde{p} \rightarrow 0$. If we take first $\tilde{p} \rightarrow 0$ then $\tilde{p}^{2}<<\frac{1}{\Lambda^{2}}$ and $\Lambda_{e f f}=\Lambda$ showing that we recover the effective commutative theory

$$
\int d^{4} p \frac{1}{2}\left(p^{2}+M^{\prime 2}\right) \phi(p) \phi(-p)
$$

If, however, we take $\Lambda \rightarrow \infty$ then $\tilde{p}^{2}>>\frac{1}{\Lambda^{2}}$ and $\Lambda_{e f f}^{2}=\frac{1}{\tilde{p}^{2}}$ and we get

$$
\int d^{4} p \frac{1}{2}\left(p^{2}+M^{2}+\frac{g^{2}}{96 \pi^{2} \tilde{p}^{2}}-\frac{g^{2} M^{2}}{96 \pi^{2}} \ln \left(\frac{1}{M^{2} \tilde{p}^{2}}\right)+\ldots\right) \phi(p) \phi(-p),
$$

which is singular when $\tilde{p} \rightarrow 0$. This shows that the limit $\Lambda \rightarrow \infty$ does not commute with the low momentum limit $\tilde{p} \rightarrow 0$ so that there is a mixing of UV and IR limits.

The theory is renormalizable at one loop order if we do not take $\tilde{p} \rightarrow 0$. What about higher loop orders? Suppose we have insertions of one loop mass corrections. Eventually we will have to integrate over small values of $\tilde{p}$ which diverges when $\Lambda \rightarrow \infty$. Then we find an IR divergence in a massive theory. This combination of UV and IR divergences makes the theory non-renormalizable.

There are also examples of noncommutative theories which are nonrenormalizable already at one loop order [i] $\overline{3}$ in. For a complex scalar field with interaction $\phi^{*} \star \phi^{*} \star \phi \star \phi$ it is found that the theory is one loop nonrenormalizable while $\phi^{*} \star \phi \star \phi^{*} \star \phi$ gives a one loop renormalizable model.

Then the main question now is the existence of a theory which is renormalizable to all loop orders. Since the UV/IR mixing appears at the level of quadratic divergences a candidate theory would be a supersymmetric one because it does not have such divergences. As we shall see this indeed happens.

\section{NONCOMMUTATIVE WESS-ZUMINO MODEL}

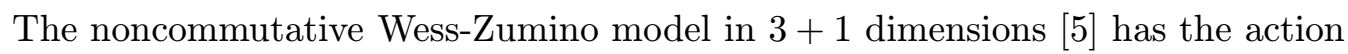




$$
\begin{aligned}
\mathcal{L}_{0}= & \frac{1}{2} \partial^{\mu} A \partial_{\mu} A+\frac{1}{2} \partial^{\mu} B \partial_{\mu} B+\frac{1}{2} \bar{\psi} i \not \partial \psi, \\
\mathcal{L}_{m}= & \frac{1}{2} F^{2}+\frac{1}{2} G^{2}+m F A+m G B-\frac{1}{2} m \bar{\psi} \psi, \\
\mathcal{L}_{g}= & g(F \star A \star A-F \star B \star B+G \star A \star B+G \star B \star A- \\
& \left.\bar{\psi} \star \psi \star A-\bar{\psi} \star i \gamma_{5} \psi \star B\right),
\end{aligned}
$$

where $A$ and $B$ are bosonic fields, $F$ and $G$ are auxiliary fields and $\psi$ is a Majorana spinor. The action is invariant under the usual supersymmetry transformations. The supersymmetry transformations are not modified by the Moyal product since they are linear in the fields. The elimination of the auxiliary fields through their equations of motion produces quartic interactions. In terms of the complex field $\phi=A+i B$ we get $\phi^{*} \star \phi^{*} \star \phi \star \phi$ which is non-renormalizable in the noncommutative case. This casts doubts about the renormalizability of the model but as we shall see supersymmetry saves the day.

As usual, the propagators are not modified by noncommutativity. They are given by

$$
\begin{aligned}
\Delta_{A A}(p) & =\Delta(p) \equiv \frac{i}{p^{2}-m^{2}+i \epsilon}, \\
\Delta_{F F}(p) & =p^{2} \Delta(p), \\
\Delta_{A F}(p) & =\Delta_{F A}(p)=-m \Delta(p), \\
S(p) & =\frac{i}{\not p-m} .
\end{aligned}
$$

Taking into account the symmetries, the vertices are

$$
\begin{array}{lll}
F A^{2} & \text { vextex: } & i g \cos \left(p_{1} \wedge p_{2}\right), \\
F B^{2} & \text { vextex: } & -i g \cos \left(p_{1} \wedge p_{2}\right), \\
G A B & \text { vertex: } & 2 i g \cos \left(p_{1} \wedge p_{2}\right), \\
\bar{\psi} \psi A & \text { vertex: } & -i g \cos \left(p_{1} \wedge p_{2}\right), \\
\bar{\psi} \psi B & \text { vertex: } & -i g \gamma_{5} \cos \left(p_{1} \wedge p_{2}\right) .
\end{array}
$$

The degree of superficial divergence for a generic 1PI graph $\gamma$ is then

$$
d(\gamma)=4-I_{A F}-I_{B F}-N_{A}-N_{B}-2 N_{F}-2 N_{G}-\frac{3}{2} N_{\psi},
$$

where $N_{\mathcal{O}}$ denotes the number of external lines associated to the field $\mathcal{O}$ and $I_{A F}$ and $I_{B F}$ are the numbers of internal lines associated to the mixed propagators $A F$ and $B F$, respectively. In all cases we will regularize the divergent Feynman integrals by assuming that a supersymmetric regularization scheme does exist.

The one loop analysis can be done in a straightforward way. As in the commutative case all tadpoles contributions add up to zero. We have verified this explicitly. The selfenergy of $A$ can be computed and the divergent part is contained in the integral

$$
16 g^{2} \int \frac{d^{4} k}{(2 \pi)^{4}}\left(1+\frac{1}{2} \cos (k \wedge p)\right) \frac{(p \cdot k)^{2}}{\left(k^{2}-m^{2}\right)^{3}} .
$$


The first term is logarithmically divergent. It differs by a factor 2 from the commutative case. As usual, this divergence is eliminated by a wave function renormalization. The second term is UV convergent and for small $p$ it behaves as $p^{2} \ln \left(p^{2} / m^{2}\right)$ and actually vanishes for $p=0$. Then there is no IR pole. The same analysis can be carried out for the others fields. For $F$ we find that the divergent part is

$$
4 g^{2} \int \frac{d^{4} k}{(2 \pi)^{4}}\left(1+\frac{1}{2} \cos (k \wedge p)\right) \frac{1}{\left(k^{2}-m^{2}\right)^{2}} .
$$

The first term is logarithmically divergent and can also be eliminated by a wave function renormalization. The second term diverges as $\ln \left(p^{2} / m^{2}\right)$ as $p$ goes to zero. However its multiple insertions is harmless. For the fermion field the divergent part is similar to the former results and needs also a wave function renormalization. The term containing $\cos (k \wedge p)$ behaves as $\not p \ln \left(p^{2} / m^{2}\right)$ and vanishes as $p$ goes to zero. Therefore, there is no $\mathrm{UV} / \mathrm{IR}$ mixing in the self-energy as expected.

To show that the model is renormalizable we must also look into the interactions vertices. The $A^{3}$ vertex has no divergent parts as in the commutative case. The same happens for the other three point functions. For the four point vertices no divergence is found as in the commutative case. Hence, the noncommutative Wess-Zumino model is renormalizable at one loop with a wave-function renormalization and no UV/IR mixing.

To go to higher loop orders we proceed as in the commutative case. We derived the supersymmetry Ward identities for the n-point vertex function. Then we showed that there is a renormalization prescription which is consistent with the Ward identities. They are the same as in the commutative case. And finally we fixed the primitively divergent vertex functions. Then we found that there is only a common wave function renormalization as in the commutative case. In general we expect

$$
\varphi_{R}=Z^{-1 / 2} \varphi, \quad m_{R}=Z m+\delta m, \quad g_{R}=Z^{3 / 2} Z^{\prime} g .
$$

At one loop we found $\delta m=0$ and $Z^{\prime}=1$. We showed that this also holds to all orders and no mass renormalization is needed.

Being the only consistent noncommutative quantum field theory in $3+1$ dimensions known so far it is natural to study it in more detail. As a first step in this direction we considered the non-relativistic limit of the noncommutative Wess-Zumino model [6i]. We found the low energy effective potential mediating the fermion-fermion and bosonboson elastic scattering in the non-relativistic regime. Since noncommutativity breaks Lorentz invariance we formulated the theory in the center of mass frame of reference where the dynamics simplifies considerably. For the fermions we found that the potential is significantly changed by the noncommutativity while no modification was found for the bosonic sector. The modifications found give rise to an anisotropic differential cross section.

Subsequently the model was formulated in superspace and again found to be renormalizable to all loop orders [1] 1 in]. The one and two loops contributions to the effective action in superspace were also found [i] $\left.{ }_{1}^{2}\right]$. The one loop Kahlerian effective potential does not get modified by noncommutativity and the two loops nonplanar contributions to the Kahlerian

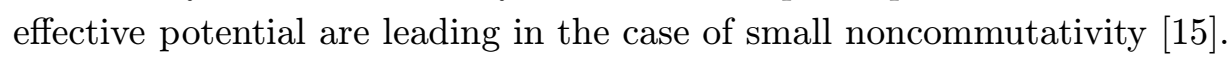




\section{Noncommutative Gross-Neveu and Nonlinear Sigma Models}

Another model where non-renormalizability is spoiled by the noncommutativity is the $O(N)$ Gross-Neveu model. The commutative model is perturbatively renormalizable in $1+1$ dimensions and $1 / N$ renormalizable in $1+1$ and $2+1$ dimensions. In both cases it presents dynamical mass generation. It is described by the Lagrangian

$$
\mathcal{L}=\frac{i}{2} \bar{\psi}_{i} \not \partial \psi_{i}+\frac{g}{4 N}\left(\bar{\psi}_{i} \psi_{i}\right)\left(\bar{\psi}_{j} \psi_{j}\right)
$$

where $\psi_{i}, i=1, \ldots N$, are two-component Majorana spinors. Since it is renormalizable in the $1 / N$ expansion in $1+1$ and $2+1$ dimensions we will consider both cases. As usual, we introduce an auxiliary field $\sigma$ and the Lagrangian turns into

$$
\mathcal{L}=\frac{i}{2} \bar{\psi}_{i} \not \partial \psi_{i}-\frac{\sigma}{2}\left(\bar{\psi}_{i} \psi_{i}\right)-\frac{N}{4 g} \sigma^{2}
$$

Replacing $\sigma$ by $\sigma+M$ where $M$ is the VEV of the original $\sigma$ we get the gap equation (in Euclidean space)

$$
\frac{M}{2 g}-\int \frac{d^{D} k}{(2 \pi)^{D}} \frac{M}{k_{E}^{2}+M^{2}}=0
$$

To eliminate the UV divergence we need to renormalize the coupling constant by

$$
\frac{1}{g}=\frac{1}{g_{R}}+2 \int \frac{d^{D} k}{(2 \pi)^{D}} \frac{1}{k_{E}^{2}+\mu^{2}}
$$

In $2+1$ dimensions we find

$$
\frac{1}{g_{R}}=\frac{\mu-|M|}{2 \pi}
$$

and therefore only for $-\frac{1}{g_{R}}+\frac{\mu}{2 \pi}>0$ it is possible to have $M \neq 0$, otherwise $M$ is necessarily zero. No such a restriction exists in $1+1$ dimensions. In any case, we will focus only in the massive phase. The propagator for $\sigma$ is proportional to the inverse of the following expression

$$
-\frac{i N}{2 g}-i N \int \frac{d^{D} k}{(2 \pi)^{D}} \frac{k \cdot(k+p)+M^{2}}{\left(k^{2}-M^{2}\right)\left[(k+p)^{2}-M^{2}\right]},
$$

which is divergent. Taking into account the gap equation the above expression reduces to

$$
\frac{\left(p^{2}-4 M^{2}\right) N}{2} \int \frac{d^{D} k}{(2 \pi)^{D}} \frac{1}{\left(k^{2}-M^{2}\right)\left[(k+p)^{2}-M^{2}\right]},
$$

which is finite. Then there is a fine tuning which is responsible for the elimination of the divergence and which might be absent in the noncommutative case due to the UV/IR mixing.

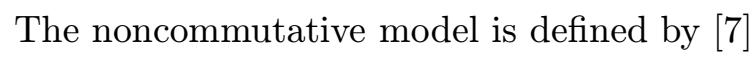

$$
S_{G N}=\int d^{D} x\left[\frac{i}{2} \bar{\psi} \not \partial \psi-\frac{M}{2} \bar{\psi} \psi-\frac{1}{2} \sigma \star(\bar{\psi} \star \psi)-\frac{N}{4 g} \sigma^{2}-\frac{N}{2 g} M \sigma\right] .
$$


Elimination of the auxiliary field results in a four-fermion interaction of the type $\bar{\psi}_{i}$ * $\psi_{i} \star \bar{\psi}_{j} \star \psi_{j}$. However a more general four-fermion interaction may involve a term like $\bar{\psi}_{i} \star \bar{\psi}_{j} \star \psi_{i} \star \psi_{j}$. This last combination does not have a simple $1 / N$ expansion and we will not consider it. The Moyal product does not affect the propagators and the trilinear vertex acquires a correction of $\cos \left(p_{1} \wedge p_{2}\right)$ with regard to the commutative case. Hence the gap equation is not modified, while the propagator for the $\sigma$ is now proportional to the inverse of

$$
-\frac{i N}{2 g}-N \int \frac{d^{D} k}{(2 \pi)^{D}} \cos ^{2}(k \wedge p) \frac{k \cdot(k+p)+M^{2}}{\left(k^{2}-M^{2}\right)\left[(k+p)^{2}-M^{2}\right]} .
$$

Now the divergent part is no longer canceled and this turns the model into a nonrenormalizable one.

On the other side, the nonlinear sigma model also presents troubles in its noncommutative version. The noncommutative model is described by

$$
\mathcal{L}=-\frac{1}{2} \varphi_{i}\left(\partial^{2}+M^{2}\right) \varphi_{i}+\frac{1}{2} \lambda \star \varphi_{i} \star \varphi_{i}-\frac{N}{2 g} \lambda,
$$

where $\varphi_{i}, i=1, \ldots, N$, are real scalar fields, $\lambda$ is the auxiliary field and $M$ is the generated mass. The leading correction to the $\varphi$ self-energy is

$$
-i \int \frac{d^{2} k}{(2 \pi)^{2}} \frac{\cos ^{2}(k \wedge p)}{(k+p)^{2}-M^{2}} \Delta_{\lambda}(k)
$$

where $\Delta_{\lambda}$ is the propagator for $\lambda$. As for the case of the scalar field this can be decomposed as a sum of a quadratically divergent part and a UV finite part. Again there is the UV/IR mixing destroying the $1 / N$ expansion.

\section{Noncommutative Supersymmetric Nonlinear Sigma Model}

The Lagrangian for the commutative supersymmetric sigma model is given by

$$
\mathcal{L}=\frac{1}{2} \partial^{\mu} \varphi_{i} \partial_{\mu} \varphi_{i}+\frac{i}{2} \bar{\psi}_{i} \not \partial \psi_{i}+\frac{1}{2} F_{i} F_{i}+\sigma \varphi_{i} F_{i}+\frac{1}{2} \lambda \varphi_{i} \varphi_{i}-\frac{1}{2} \sigma \bar{\psi}_{i} \psi_{i}-\bar{\xi} \psi_{i} \varphi_{i}-\frac{N}{2 g} \lambda,
$$

where $F_{i}, i=1, \ldots, N$, are auxiliary fields. Furthermore, $\sigma, \lambda$ and $\xi$ are the Lagrange multipliers which implement the supersymmetric constraints. After the change of variables $\lambda \rightarrow \lambda+2 M \sigma, F \rightarrow F-M \varphi$ where $M=\left\langle\sigma>\right.$, and the shifts $\sigma \rightarrow \sigma+M$ and $\lambda \rightarrow \lambda+\lambda_{0}$, where $\lambda_{0}=\langle\lambda\rangle$, we arrive at a more symmetric form for the Lagrangian

$$
\begin{aligned}
\mathcal{L}= & -\frac{1}{2} \varphi_{i}\left(\partial^{2}+M^{2}\right) \varphi_{i}+\frac{1}{2} \bar{\psi}_{i}(i \not \partial-M) \psi_{i}+\frac{1}{2} F_{i}^{2}+M^{2} \varphi_{i}^{2}+\frac{1}{2} \lambda_{0} \varphi_{i}^{2} \\
& +\frac{1}{2} \lambda \varphi_{i}^{2}+\sigma \varphi_{i} F_{i}-\frac{1}{2} \sigma \bar{\psi}_{i} \psi_{i}-\bar{\xi} \psi_{i} \varphi_{i}-\frac{N}{2 g} \lambda-\frac{N}{g} M \sigma .
\end{aligned}
$$

Now supersymmetry requires $\lambda_{0}=-2 M^{2}$ and the gap equation is

$$
\int \frac{d^{D} k}{(2 \pi)^{D}} \frac{i}{k^{2}-M^{2}}=\frac{1}{g}
$$


so a coupling constant renormalization is required. We now must examine whether the propagator for $\sigma$ depends on the this renormalization. We find that the two point function for $\sigma$ is proportional to the inverse of

$$
\frac{\left(p^{2}-4 M^{2}\right) N}{2} \int \frac{d^{D} k}{(2 \pi)^{D}} \frac{1}{\left(k^{2}-M^{2}\right)\left[(k+p)^{2}-M^{2}\right]},
$$

which is identical to the Gross-Neveu case. Notice that the gap equation was not used. The finiteness of the above expression is a consequence of supersymmetry.

The noncommutative version of the supersymmetric nonlinear sigma model is given by [i]i]

$$
\begin{aligned}
\mathcal{L}= & -\frac{1}{2} \varphi_{i}\left(\partial^{2}+M^{2}\right) \varphi_{i}+\frac{1}{2} \bar{\psi}_{i}(i \not \partial-M) \psi_{i}+\frac{1}{2} F_{i}^{2}+\frac{\lambda}{2} \star \varphi_{i} \star \varphi_{i} \\
& -\frac{1}{2} F_{i} \star\left(\sigma \star \varphi_{i}+\varphi_{i} \star \sigma\right)-\frac{1}{2} \sigma \star \bar{\psi}_{i} \star \psi_{i}-\frac{1}{2}\left(\bar{\xi} \star \psi_{i} \star \varphi_{i}+\bar{\xi} \star \varphi_{i} \star \psi_{i}\right) \\
& -\frac{N}{2 g} \lambda-\frac{N M \sigma}{g} .
\end{aligned}
$$

Notice that supersymmetry dictates the form of the trilinear vertices. Also, the supersymmetry transformations are not modified by noncommutativity since they are linear and no Moyal products are required.

The propagators are the same as in the commutative case. The vertices have cosine factors due to the Moyal product

$$
\begin{array}{ccl}
\lambda \varphi^{2} & \text { vertex: } & \frac{i}{2} \cos \left(p_{1} \wedge p_{2}\right), \\
\sigma \varphi F & \text { vertex: } & -i \cos \left(p_{1} \wedge p_{2}\right), \\
\bar{\psi} \psi \sigma & \text { vertex: } & -\frac{i}{2} \cos \left(p_{1} \wedge p_{2}\right) \\
\bar{\xi} \psi \varphi & \text { vertex: } & -i \cos \left(p_{1} \wedge p_{2}\right) .
\end{array}
$$

We again consider the propagators for the Lagrange multiplier fields. Now the $\sigma$ propagator is modified by the cosine factors and is proportional to the inverse of

$$
\frac{\left(p^{2}-4 M^{2}\right) N}{2} \int \frac{d^{D} k}{(2 \pi)^{D}} \frac{\cos ^{2}(k \wedge p)}{\left(k^{2}-M^{2}\right)\left[(k+p)^{2}-M^{2}\right]} .
$$

It is well behaved both in UV and IR regions. The propagators for $\lambda$ and $\xi$ are proportional to the inverse of

$$
\frac{N}{2} \int \frac{d^{D} k}{(2 \pi)^{D}} \cos ^{2}(k \wedge p) \frac{1}{\left[(k+p)^{2}-M^{2}\right]\left[k^{2}-M^{2}\right]},
$$

and

$$
N \frac{(\not p+2 M)}{2} \int \frac{d^{D} k}{(2 \pi)^{D}} \cos ^{2}(k \wedge p) \frac{1}{\left[(k+p)^{2}-M^{2}\right]\left[k^{2}-M^{2}\right]},
$$

respectively. They are also well behaved in UV and IR regions. 
The degree of superficial divergence for a generic 1PI graph $\gamma$ is

$$
d(\gamma)=D-\frac{(D-1)}{2} N_{\psi}-\frac{(D-2)}{2} N_{\varphi}-\frac{D}{2} N_{F}-N_{\sigma}-\frac{3}{2} N_{\xi}-2 N_{\lambda},
$$

where $N_{\mathcal{O}}$ is the number of external lines associated to the field $\mathcal{O}$. Potentially dangerous diagrams are those contributing to the self-energies of the $\varphi$ and $\psi$ fields since, in principle, they are quadratic and linearly divergent, respectively. For the self-energies of $\varphi$ and $\psi$ we find that they diverge logarithmically and they can be removed by a wave function renormalization of the respective field. The same happens for the auxiliary field $F$. The renormalization factors for them are the same so supersymmetry is preserved in the noncommutative theory. This analysis can be extended to the n-point functions. In $2+1$ dimensions we find nothing new showing the renormalizability of the model at leading order of $1 / N$. However, in $1+1$ dimensions there some peculiarities. Since the scalar field is dimensionless in $1+1$ dimensions any graph involving an arbitrary number of external $\varphi$ lines is quadratically divergent. In the four-point function there is a partial cancellation of divergences but a logarithmic divergence still survives. The counterterm needed to remove it can not be written in terms of $\int d^{2} x \varphi_{i} \star \varphi_{i} \star \varphi_{j} \star \varphi_{j}$ and $\int d^{2} x \varphi_{i} \star \varphi_{j} \star \varphi_{i} \star \varphi_{j}$. A possible way to remove this divergence is by generalizing the definition of 1PI diagram. However the cosine factors do not allow us to use this mechanism which casts doubt about the renormalizability of the noncommutative supersymmetric $O(N)$ nonlinear sigma model in $1+1$ dimensions.

The noncommutative supersymmetric nonlinear sigma model can also be formulated in superspace [i, $\overline{\overline{6}} \overline{1}$. There it is possible to go beyond the sub-leading order in $1 / N$. It is then possible to show that model is renormalizable to all orders of $1 / \mathrm{N}$ and explicitly verify that it is asymptotically free [i] $\left.{ }^{1} \overline{\underline{6}}\right]$.

\section{Spontaneous Symmetry Breaking in Noncommutative Field Theory}

Having seen the important role supersymmetry plays in noncommutative models it is natural to go further. Spontaneous symmetry breaking and the Goldstone theorem are essential in the standard model and the effect of noncommutativity in this setting deserves to be fully understood. In four dimensions it is known that spontaneous symmetry breaking can occur for the $U(N)$ model but not for the $O(N)$ unless $N=2$. The $O(2)$ case was analyzed

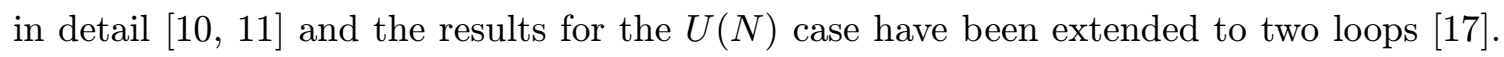
Going to higher loops requires an IR regulator which can no longer be removed[1] to these troubles we will consider three dimensional models.

Let us consider the three-dimensional action [i:

$$
\begin{aligned}
S & =\int d^{3} x\left[-\frac{1}{2} \phi_{a} \square \phi_{a}+\frac{\mu^{2}}{2} \phi_{a} \phi_{a}\right. \\
& -\frac{g}{4}\left(l_{1} \phi_{a} * \phi_{a} * \phi_{b} * \phi_{b}+l_{2} \phi_{a} * \phi_{b} * \phi_{a} * \phi_{b}\right) \\
& -\frac{\lambda}{6}\left(h_{1} \phi_{a} * \phi_{a} * \phi_{b} * \phi_{b} * \phi_{c} * \phi_{c}+h_{2} \phi_{a} * \phi_{a} * \phi_{b} * \phi_{c} * \phi_{c} * \phi_{b}+\right. \\
& +h_{3} \phi_{a} * \phi_{a} * \phi_{b} * \phi_{c} * \phi_{b} * \phi_{c}+h_{4} \phi_{a} * \phi_{b} * \phi_{c} * \phi_{a} * \phi_{b} * \phi_{c}+
\end{aligned}
$$




$$
\left.\left.+h_{5} \phi_{a} * \phi_{b} * \phi_{c} * \phi_{a} * \phi_{c} * \phi_{b}\right)\right]
$$

where $l_{1}, l_{2}, h_{1}, h_{2} \ldots h_{5}$ are real numbers satisfying the conditions $l_{1}+l_{2}=1$ and $h_{1}+$ $h_{2}+\ldots+h_{5}=1$, so that there are two quartic and five sextuple independent interaction couplings. The potential has a minimum for $\phi_{a} \phi_{a}=a^{2}$ with

$$
a^{2}=\frac{1}{2 \lambda}\left(-g+\sqrt{g^{2}+4 \mu^{2} \lambda}\right) .
$$

As usual we introduce the field $\pi_{i}$ and $\sigma$ having vanishing expectation value. The action then becomes

$$
\begin{aligned}
S & =\int d^{3} x\left\{-\frac{1}{2} \pi_{i} \square \pi_{i}-\frac{1}{2} \sigma\left(\square-m^{2}\right) \sigma-\left(2 \lambda a^{3}+g a\right) \sigma * \pi_{i} * \pi_{i}-\left(\frac{10}{3} \lambda a^{3}+g a\right) \sigma * \sigma * \sigma\right. \\
& -\left[\left(\frac{\lambda}{6} \alpha a^{2}+\frac{g}{4} l_{1}\right) \pi_{i} * \pi_{i} * \pi_{j} * \pi_{j}+\left(\frac{\lambda}{6}(3-\alpha) a^{2}+\frac{g}{4} l_{2}\right) \pi_{i} * \pi_{j} * \pi_{i} * \pi_{j}\right. \\
& +\left(\frac{\lambda}{6} a^{2} \beta+\frac{g}{2} l_{1}\right) \sigma * \sigma * \pi_{i} * \pi_{i}+\left(\frac{\lambda}{6} a^{2}(18-\beta)+\frac{g}{2} l_{2}\right) \sigma * \pi_{i} * \sigma * \pi_{i} \\
& \left.\left.++\left(\frac{5}{2} \lambda a^{2}+\frac{g}{4}\right) \sigma * \sigma * \sigma * \sigma\right]+\ldots\right\},
\end{aligned}
$$

where $m^{2}=4 \mu^{2}-2 g a^{2}=4 \lambda a^{4}+2 g a^{2}$, the dots denote terms of fifth and sixth order in the fields, $\alpha=3 h_{1}+2\left(h_{2}+h_{3}\right)+h_{5}$, and $\beta=18 h_{1}+14 h_{2}+12 h_{3}+6 h_{4}+8 h_{5}$. Notice that condition ( $(\overline{6} .2 i)$ implies that the pions are massless in the tree approximation, in agreement with the Goldstone theorem.

We then find the propagators

$$
\begin{aligned}
& <\sigma\left(p_{1}\right) \sigma\left(p_{2}\right)>=(2 \pi)^{d} \delta\left(p_{1}+p_{2}\right) \frac{i}{p_{1}^{2}-m^{2}}, \\
& <\pi_{i}\left(p_{1}\right) \pi_{j}\left(p_{2}\right)>=(2 \pi)^{d} \delta\left(p_{1}+p_{2}\right) \frac{i \delta_{i j}}{p_{1}^{2}}
\end{aligned}
$$

and the vertices present the usual phase factors

$$
\begin{aligned}
& \pi_{i}\left(p_{1}\right) \pi_{j}\left(p_{2}\right) \pi_{k}\left(p_{3}\right) \pi_{l}\left(p_{4}\right) \rightarrow-i \rho_{1}\left[\cos \left(p_{1} \wedge p_{2}\right) \cos \left(p_{3} \wedge p_{4}\right) \delta_{i j} \delta_{k l}\right. \\
& \left.+\cos \left(p_{1} \wedge p_{3}\right) \cos \left(p_{2} \wedge p_{4}\right) \delta_{i k} \delta_{j l}+\cos \left(p_{1} \wedge p_{4}\right) \cos \left(p_{2} \wedge p_{3}\right) \delta_{i l} \delta_{k j}\right] \\
- & i \rho_{2}\left[\cos \left(p_{1} \wedge p_{3}+p_{2} \wedge p_{4}\right) \delta_{i j} \delta_{k l}+\cos \left(p_{1} \wedge p_{2}+p_{3} \wedge p_{4}\right)\right] \delta_{i k} \delta_{j l} \\
& \left.+\cos \left(p_{1} \wedge p_{2}+p_{4} \wedge p_{3}\right) \delta_{i l} \delta_{k j}\right], \\
& \pi_{j}\left(p_{1}\right) \pi_{j}\left(p_{2}\right) \sigma\left(p_{3}\right) \sigma\left(p_{4}\right) \rightarrow-i\left[\rho_{3} \cos \left(p_{1} \wedge p_{2}\right) \cos \left(p_{3} \wedge p_{4}\right)+\right. \\
+ & \left.\rho_{4} \cos \left(p_{1} \wedge p_{3}+p_{2} \wedge p_{4}\right)\right], \\
& \sigma\left(p_{1}\right) * \pi_{i}\left(p_{2}\right) * \pi_{i}\left(p_{3}\right) \rightarrow-i\left(4 \lambda a^{3}+2 g a\right) \cos \left(p_{2} \wedge p_{3}\right),
\end{aligned}
$$

where $\rho_{1}=\frac{4 \lambda}{3} a^{2} \alpha+2 g l_{1}, \rho_{2}=(3-\alpha) \frac{4 \lambda}{3} a^{2}+2 g l_{2}, \rho_{3}=\frac{2 \lambda}{3} a^{2} \beta+2 g l_{1}$ and $\rho_{4}=\frac{2 \lambda}{3} a^{2}(18-$ $\beta)+2 g l_{2}$.

The gap equation receives no contribution from noncommutativity while the one loop corrections to the pion mass have divergences both, in the planar and non-planar sectors. 
Eliminating the UV divergence in the planar sector also eliminates the UV/IR mixing in the non-planar sector. It is also fortunate that it leads to an analytic behavior in the IR so that the mass corrections vanish for $p=0$. This mechanism does not appears in the four dimensional case.

The two point function for $\sigma$ is also analytic in the IR leading to a relation among the parameters. The divergences in the higher point functions can also be eliminated. Therefore, we have shown that this $O(N)$ model is renormalizable at one loop for any $N$ [8], in contradistinction to the four dimensional case where $N$ must be equal to 2 .

A supersymmetric version of this model can be formulated in superspace. Again, the gap equation is not affected by noncommutativity. The mass corrections for the pion two point function are UV finite and free of UV/IR mixing as expected. It also vanishes for $p=0$. Supersymmetry does not appear to be important in this situation.

\section{Conclusions}

We have shown that it is possible to build consistent quantum field theories in noncommutative space. Supersymmetry is an essential ingredient for renormalizability. The models studied here do not involve gauge fields and this considerably simplifies the situation. All vertices are deformed in the same way by the Moyal product and this was essential to analyze the amplitudes. With gauge fields the situation is much more complicated because the vertices are deformed in different ways. However, supersymmetric gauge theories may still have a better behavior. The analysis of spontaneous symmetry breaking in three dimensions revealed that it is possible to implement the Goldstone theorem in noncommutative theories. Supersymmetry seems to play no essential role in this case.

\section{Acknowledgments}

This work was done in collaboration with H. O. Girotti, M. Gomes, A. J. da Silva and A. Petrov. It was partially supported by Conselho Nacional de Desenvolvimento Científico e Tecnológico (CNPq), and PRONEX under contract CNPq 66.2002/1998-99.

\section{References}

[1] For a review see: R. Szabo, "Quantum field theory on noncommutative spaces", hep-th/0109162; N.A. Nekrasov and M. Douglas, "Noncommutative field theory", hep-th/0106048, Rev. Mod. Phys. 73, 977, 2002; V. O. Rivelles, "Noncommutative supersymmetric field theories", hep-th/0103131, Braz. J. Phys. 31, 2552001.

[2] S. Minwalla and M. van Raamsdonk, N. Seiberg, "Noncommutative perturbative dynamics", hep-th/9912072, JHEP 0002, 020, 2000.

[3] F. Ruiz Ruiz, "Gauge fixing independence of IR divergences in noncommutative U(1), perturbative tachyonic instabilities and supersymmetry", hep-th/0012171, Phys. Lett. B502 2742001 .

[4] A. Matusis, L.Susskind and N. Toumbas, "The IR/UV connection in the noncommutative gauge theories", hep-th/0002075, JHEP 00120022000. 
[5] H.O. Girotti, M. Gomes, V.O. Rivelles and A.J. da Silva, 'A consistent noncommutative field theory: The Wess-Zumino model", hep-th/0005272, Nucl. Phys. B587, 299, 2000.

[6] H. O. Girotti, M. Gomes, V. O. Rivelles, A. J. da Silva, "The Low Energy Limit of the Noncommutative Wess-Zumino Model", hep-th/0101159, JHEP 0205 (2002) 040.

[7] H. O. Girotti, M. Gomes, V. O. Rivelles, A. J. da Silva, "The Noncommutative Supersymmetric Nonlinear Sigma Model", hep-th/0102101, Int. J. Mod. Phys. A 17 (2002) 1503.

[8] H. O. Girotti, M. Gomes, A. Y. Petrov, V. O. Rivelles and A. J. da Silva, "Spontaneous symmetry breaking in noncommutative field theory," hep-th/0207220.

[9] B. A. Campbell and K. Kaminsky, "Noncommutative field theory and spontaneous symmetry breaking", hep-th/0003137, Nucl. Phys. B 581, 240 (2000); "Noncommutative linear sigma models", hep-th/0102022, Nucl. Phys. B 606, 613 (2001).

[10] F. J. Petriello, "The Higgs mechanism in noncommutative gauge theories", hep-th/0101109, Nucl. Phys. B 601, 169 (2001).

[11] F. Ruiz Ruiz, "UV/IR mixing and the Goldstone theorem in noncommutative field theory", hep-th/0202011.

[12] T. Filk, "Divergences In A Field Theory On Quantum Space," Phys. Lett. B 376 (1996) 53.

[13] I. Y. Aref'eva, D. M. Belov and A. S. Koshelev, "A note on UV/IR for Noncommutative Complex Scalar Field", hep-th/0001215.

[14] A. A. Bichl, J. M. Grimstrup, H. Grosse, L. Popp, M. Schweda and R. Wulkenhaar, "The superfield formalism applied to the noncommutative Wess-Zumino model," JHEP 0010 (2000) 046, hep-th/0007050.

[15] I. L. Buchbinder, M. Gomes, A. Y. Petrov and V. O. Rivelles, "Superfield effective action in the noncommutative Wess-Zumino model", hep-th/0107022, Phys. Lett. B 517, 191 (2001).

[16] H. O. Girotti, M. Gomes, A. Y. Petrov, V. O. Rivelles and A. J. da Silva, "The three-dimensional noncommutative nonlinear sigma model in superspace", hep-th/0109222, Phys. Lett. B 521, 119 (2001).

[17] Y. Liao, "Validity of Goldstone theorem at two loops in noncommutative U(N) linear sigma model", hep-th/0204032.

[18] S. Sarkar and B. Sathiapalan, "Comments on the renormalizability of the broken symmetry phase in noncommutative scalar field theory" , hep-th/0104106, JHEP 0105, 049 (2001); S. Sarkar, "On the UV renormalizability of noncommutative field theories", JHEP 0206, 003 (2002), hep-th/0202171. 